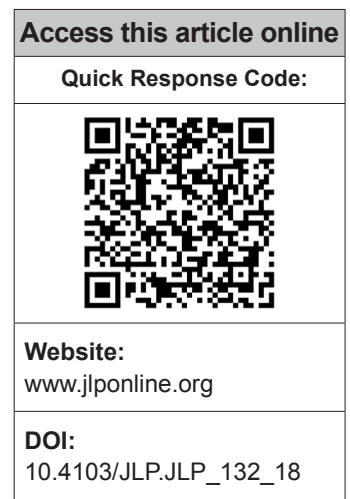

Department of Microbiology, JSS Medical College, JSS Academy of Higher Education and Research, Mysore, Karnataka, India

Address for correspondence: Dr. Ranjitha S. Gowda, Department of Microbiology, JSS Medical College, Shivarathreswara Nagar, Mysore - 570 015, Karnataka, India E-mail: ranju.vish85@ gmail.com

Submission: 07-10-2018 Accepted: 03-04-2019

\title{
Detection of carbapenemase production in Enterobacteriaceae and Pseudomonas species by carbapenemase Nordmann-Poirel test
}

\author{
Morubagal R. Rao, Pooja Chandrashaker, Rashmi P. Mahale, Sowmya G. Shivappa, \\ Ranjitha S. Gowda, Vidyavathi B. Chitharagi
}

\begin{abstract}
:
PURPOSE: Multidrug-resistant organisms causing community-acquired and hospital-acquired infections are increasing at a dangerous rate. Carbapenemase-producing Enterobacteriaceae and Pseudomonas species are an important source of concern since these organisms are not only resistant to beta-lactam antibiotics but also show cross-resistance to other groups of antibiotics. In the present study, rapid detection of these carbapenemase-producing Enterobacteriaceae and Pseudomonas species by carbapenemase Nordmann-Poirel (Carba NP) test was evaluated by comparing with modified Hodge test (MHT).
\end{abstract}

MATERIALS AND METHODS: Imipenem-resistant Enterobacteriaceae and Pseudomonas species isolated from various samples such as pus, blood, sputum, urine, and endotracheal aspirates were processed for carbapenemase detection by MHT and Carba NP test. Kappa analysis was done to evaluate the percentage agreement between the two tests.

RESULTS: Seventy imipenem-resistant Enterobacteriaceae and Pseudomonas isolates were analyzed in the present study for carbapenemase production. $63.41 \%$ of Enterobacteriaceae and $34.48 \%$ of Pseudomonas species were carbapenemase producers considering both the methods. By $\mathrm{MHT}, 36(51.42 \%)$ isolates and, by Carba NP test, $35(50 \%)$ isolates were positive for carbapenemase production out of the 70 isolates.

CONCLUSION: Carba NP test when compared to MHT is a simple, rapid, cost-effective biochemical test which can be used in all laboratories in the identification of life-threatening carbapenemase-producing Gram-negative bacteria.

Key words:

Carbapenemase, carbapenemase Nordmann-Poirel, Enterobacteriaceae, modified Hodge test, Pseudomonas

\section{Introduction}

$\mathrm{M}$ ultidrug-resistant organisms causing community-acquired and hospital-acquired infections are increasing at a dangerous rate globally, especially among Enterobacteriaceae and nonfermenters. ${ }^{[1]}$ Extended-spectrum $\beta$-lactamase (ESBL)- and acquired

This is an open access journal, and articles are distributed under the terms of the Creative Commons Attribution-NonCommercial-ShareAlike 4.0 License, which allows others to remix, tweak, and build upon the work non-commercially, as long as appropriate credit is given and the new creations are licensed under the identical terms.

For reprints contact: reprints@medknow.com cephalosporinase (AmpC)-producing organisms are resistant to almost all $\beta$-lactams with the exception of carbapenems. Treatment of choice for these ESBL- or AmpC-producing isolates is carbapenems. ${ }^{[2]}$ Therefore, it is important to preserve the clinical efficacy of carbapenems (imipenem, ertapenem, meropenem, and doripenem). However, in recent times, there is an increase in

How to cite this article: Rao MR, Chandrashaker $P$, Mahale RP, Shivappa SG, Gowda RS, Chitharagi VB. Detection of carbapenemase production in Enterobacteriaceae and Pseudomonas species by carbapenemase Nordmann-Poirel test. J Lab Physicians 2019;11:107-10. 
reporting of carbapenem-resistant Enterobacteriaceae worldwide, probably as a result of carbapenemase gene acquisition ${ }^{[3]}$ resulting in treatment failure due to carbapenem usage. Various mechanisms of carbapenem resistance in Enterobacteriaceae are due to a decrease in bacterial outer-membrane permeability, with excess production of $\beta$-lactamases with no carbapenemase activity or expression of carbapenemases. ${ }^{[4,5]}$ Klebsiella pneumoniae carbapenemase (Ambler Class A); Verona integron-encoded metallo- $\beta$-lactamase, imipenemase, and New Delhi metallo- $\beta$-lactamase (all Ambler Class B); and oxacillinase-48 (Ambler class D) are some examples of carbapenemases reported in Enterobacteriaceae. ${ }^{[6]}$

Due to other resistance mechanisms, most of the times carbapenemase-producing Enterobacteriaceae and Pseudomonas species show resistance to other groups of drugs also leading to multidrug-resistant or pandrug-resistant isolates. Rampant spread of such isolates is an important source of concern globally. ${ }^{[7]}$ This shows that for the selection of appropriate therapeutic schemes and the implementation of infection control measures, detection of carbapenemase producers is important. ${ }^{[8,9]}$ Rapid identification of carbapenemase is the need of today's clinical practice. Ultraviolet spectrophotometry, matrix-assisted laser desorption ionization-time of flight technique, and molecular methods are few examples of techniques available for rapid detection. Even though these methods have good sensitivity and specificity, they require trained microbiologists and expensive equipment. Molecular methods which are considered as gold standard method may fail to detect unknown carbapenemase genes not included in gene panel. ${ }^{[10]}$ To overcome all these drawbacks, a biochemical test (carbapenemase Nordmann-Poirel [Carba NP] test) based on a technique designed to identify the hydrolysis of the $\beta$-lactam ring of a carbapenem has been developed. ${ }^{[11]}$ The present study was undertaken to evaluate Carba NP test in discriminating carbapenemase producers from nonproducers by comparing with modified Hodge test (MHT).

\section{Materials and Methods}

This study was conducted in the department of microbiology of a teaching hospital. Ethical clearance certificate was obtained from the Institutional Ethical Committee. Various samples such as pus, blood, sputum, urine, and endotracheal aspirates received in the laboratory were inoculated on a sterility-checked MacConkey agar and blood agar plates and incubated at $37^{\circ} \mathrm{C}$ for $18-24 \mathrm{~h}$. Based on the growth on MacConkey agar and blood agar, isolates were further processed in VITEK 2 systems, for identification and antimicrobial susceptibility. Imipenem-resistant Enterobacteriaceae and Pseudomonas species were further processed for carbapenemase production by MHT and Carba NP test. Kappa analysis was done to evaluate the percentage agreement between MHT and Carba NP tests.

\section{Modified Hodge test}

Lawn culture of ATCC Escherichia coli 25922 at a turbidity equivalent to that of $0.5 \mathrm{McF}$ arland was made onto the Mueller-Hinton agar plate. After drying, an imipenem $(10 \mu \mathrm{g})$ disc was placed at the center of the plate. The test strain and control strains (a known carbapenemase-producing Pseudomonas aeruginosa was used as positive control, and E. coli ATCC 25922 was used as negative control) were heavily streaked from the edge of the imipenem disc to the periphery of the plate in different directions. The plates were incubated at $37^{\circ} \mathrm{C}$ for $18-24 \mathrm{~h}$.

\section{Interpretation}

The presence of a cloverleaf type of zone of inhibition near the test/positive control organism was interpreted as MHT positive. ${ }^{[12]}$

\section{Carbapenemase Nordmann-Poirel test}

The Carba NP test for Enterobacteriaceae and Pseudomonas spp. was performed as follows:

Two 1.5-ml low-bind protein microcentrifuge tubes (Eppendorf), each containing $100 \mu \mathrm{l}$ of a $20-\mathrm{mM}$ Tris-HCL lysis buffer, were individually inoculated with a $1-\mu 1$ loopful of bacterial colony (18-24 h old, loop swept through pure culture), and bacterial suspensions were vortexed for $5 \mathrm{~min}$. To the first tube, $100 \mu \mathrm{l}$ of $0.5 \%$ (wt/vol) phenol red solution with $10-\mathrm{mM}$ zinc sulfate (solution A, buffered to $\mathrm{pH} 7.8$ by adding $0.1 \mathrm{~N} \mathrm{NaOH}$ ) was then added, and the tube was vortexed. To the second tube, $100 \mu \mathrm{l}$ of solution A with imipenem dissolved directly in solution A to a final concentration of $6 \mathrm{mg} / \mathrm{ml}$ was added and then vortexed. A mixture of the phenol red solution and the enzymatic suspension being tested was incubated at $37^{\circ} \mathrm{C}$ for a maximum of $2 \mathrm{~h} \cdot{ }^{[13]}$ A known carbapenemase-producing $P$. aeruginosa was used as positive control, and E. coli ATCC 25922 was used as negative control.

After incubation, the presence of any carbapenemase, which hydrolyzes imipenem into its carboxylic form, leading to a $\mathrm{pH}$ decrease, was detected by a color change of phenol red solution (red to yellow/orange), while tubes remain red in the absence of carbapenemase.

\section{Interpretation}

1. First tube and second tube remaining red - noncarbapenemase-producing isolate

2. First tube remaining red and second tube turning yellow / orange - carbapenemase-producing isolate. 


\section{Results}

Seventy imipenem-resistant Enterobacteriaceae (19 - Klebsiella species, 15 - E. coli, 3 - Enterobacter cloacae, 2 - Morganella morganii, and 2 - Serratia species) and Pseudomonas species (29) isolates were analyzed in the present study.

Out of 29 Pseudomonas species, 14 were from pus samples followed by 12 from endotracheal aspirates. Out 19 Klebsiella species, 8 were from pus and 7 from urine. Sample-wise distribution of isolates is shown in Table 1.

Out of 29 imipenem-resistant Pseudomonas species studied, $17(58.6 \%)$ isolates were resistant to meropenem and $18(62.0 \%)$ isolates were resistant to doripenem. Twenty-seven (93.1\%) isolates were found to be sensitive to colistin.

Of the 19 Klebsiella species studied, total resistance was observed to imipenem and meropenem. Sixteen $(84.2 \%)$ isolates were found resistant to ertapenem. Thirteen $(68.4 \%)$ isolates were resistant to tigecycline and $15(78.94 \%)$ isolates were sensitive to colistin. Of the 15 E. coli studied, total resistance was observed to imipenem and meropenem. Ten $(71.4 \%)$ were sensitive to tigecycline and $13(92.8 \%)$ isolates were found to be sensitive to colistin.

Out of 70 isolates analyzed for carbapenemase production, $36(51.42 \%)$ isolates were carbapenemase producers by MHT and $35(50 \%)$ isolates were detected as carbapenemase producers by Carba NP test. Out of 29 pseudomonas species, 10 (34.48\%) isolates were detected as carbapenemase producer by MHT. Out of these 10 isolates, one isolate gave negative result for carbapenemase production by Carba NP test.

Among 41 Enterobacteriaceae, 26 (63.41\%) isolates were detected as carbapenemase producers. Out of 19 Klebsiella species, 14 (73.68\%) and, out of 15 E. coli, $10(66.66 \%)$ were detected as carbapenemase producers by both MHT and Carba NP test, respectively. One Morganella out of 2 and 1 Enterobacter species out of 3 were carbapenemase producers by both the methods. Both Serratia species were negative for carbapenemase production by both the methods. Kappa analysis

Table 1: Sample-wise distribution of clinical isolates

\begin{tabular}{lccccc}
\hline Organism & \multicolumn{5}{c}{$\begin{array}{c}\text { Pus Endotracheal Urine Blood Sputum } \\
\text { aspirates }\end{array}$} \\
\hline Pseudomonas species (29) & 14 & 12 & 0 & 0 & 3 \\
Klebsiella species (19) & 5 & 10 & 2 & 2 & 0 \\
Escherichia coli (15) & 8 & 0 & 7 & 0 & 0 \\
Enterobacter cloacae (3) & 2 & 0 & 0 & 1 & 0 \\
Serratia marcescens (2) & 0 & 2 & 0 & 0 & 0 \\
Morganella morganii (2) & 2 & 0 & 0 & 0 & 0 \\
\hline
\end{tabular}

revealed that the strength of agreement between the two tests is considered very good (kappa $=97 \%$, confidence interval $=0.916-1.00$ ).

\section{Discussion}

One of the greatest advances of modern medicine is the development of antibiotics for the treatment of infectious disease. Unfortunately, effectiveness of many antimicrobial agents is under threat due to the emergence of antibiotic resistance among bacteria. In order to control the emergence of drug resistance, the irrational use of antibiotics should be controlled. One of the ways of controlling antibiotic misuse is rapid detection and reporting of drug resistance mechanisms in clinical isolates, which helps in the selection of appropriate antibiotics for treatment. Keeping this in mind, the present study evaluated Carba NP test for rapid detection of carbapenemase among Enterobacteriaceae and Pseudomonas species.

A total of 70 clinical isolates (29 Pseudomonas and 41 Enterobacteriaceae) were analyzed for carbapenemase production. $63.41 \%$ of Enterobacteriaceae were carbapenemase producers $(66.66 \%$ of E. coli and $73.68 \%$ of Klebsiella species). Chauhan et al. ${ }^{[14]}$ reported $87.01 \%$ of E. coli and $91.51 \%$ of Klebsiella spp. as carbapenemase producers by MHT. These findings suggest that carbapenemase production is on rise in Enterobacteriaceae, particularly in Klebsiella species. $34.48 \%$ Pseudomonas species were detected as carbapenemase producers, and similar findings were observed in a study done by ElMasry et al., ${ }^{[15]}$ in which $37 \%$ of Pseudomonas species were reported as carbapenemase producers by polymerase chain reaction. However, by MHT, positivity was increased to $48.1 \%$.

One Pseudomonas aeruginosa strain in the present study was positive for carbapenemase production by MHT but negative by Carba NP test. Several studies have shown that GES-type carbapenemase-producing Pseudomonas species may not be detected by Carba NP test. ${ }^{[16]}$ This could be one of the reasons in the present study for Carba NP test showing negative results. However, in this study, molecular analysis was not performed to comment on sensitivity or specificity of Carba NP test.

In the present study, MHT test was performed using imipenem disc instead of ertapenem or meropenem disc because we were able to reproduce and interpret results better using imipenem disc than meropenem. Even though both Carba NP and MHT detected carbapenemase producers almost equally except for one strain which gave negative results with Carba NP test, MHT at times was difficult to interpret. 


\section{Conclusion}

Carbapenemase producing Gram-negative bacteria causing community-acquired and hospital-acquired infections are increasing at a dangerous rate globally. Methods for rapid identification of these organisms is of utmost importance. One such method is Carba NP test. When compared to MHT, Carba NP test is a simple, rapid, cost-effective biochemical test which can be used in all laboratories in the identification of life-threatening carbapenemase-producing Gram-negative bacteria.

\section{Financial support and sponsorship}

Nil.

\section{Conflicts of interest}

There are no conflicts of interest.

\section{References}

1. Nordmann P. Carriage and laboratory detection of multi-drug resistant bacteria in low and middle-income countries. Int J Infect Dis 2014;21:64.

2. Infectious Diseases Society of America (IDSA), Spellberg B, Blaser M, Guidos RJ, Boucher HW, Bradley JS. Combating antimicrobial resistance: Policy recommendations to save lives. Clin Infect Dis 2011;52 Suppl 5:S397-428.

3. Nordmann P, Dortet L, Poirel L. Carbapenem resistance in Enterobacteriaceae: Here is the storm! Trends Mol Med 2012;18:263-72.

4. Martínez-Martínez L. Extended-spectrum beta-lactamases and the permeability barrier. Clin Microbiol Infect 2008;14 Suppl 1:82-9.

5. Thomson KS. Extended-spectrum-beta-lactamase, AmpC, and carbapenemase issues. J Clin Microbiol 2010;48:1019-25.
6. Nordmann P, Naas T, Poirel L. Global spread of carbapenemase-producing Enterobacteriaceae. Emerg Infect Dis 2011;17:1791-8.

7. Nordmann P, Poirel L. Strategies for identification of carbapenemase-producing Enterobacteriaceae. J Antimicrob Chemother 2013;68:487-9.

8. Miriagou V, Cornaglia G, Edelstein M, Galani I, Giske CG, Gniadkowski M, et al. Acquired carbapenemases in gram-negative bacterial pathogens: Detection and surveillance issues. Clin Microbiol Infect 2010;16:112-22.

9. Nordmann P, Gniadkowski M, Giske CG, Poirel L, Woodford N, Miriagou V. Identification and screening of carbapenemase-producing Enterobacteriaceae. Clin Microbiol Infect 2012;18:432-8.

10. Poirel L, Nordmann P. Rapidec carba NP test for rapid detection of carbapenemase producers. J Clin Microbiol 2015;53:3003-8.

11. Nordmann P, Poirel L, Dortet L. Rapid detection of carbapenemase-producing Enterobacteriacea. Emerg Infect Dis 2012;18:1503-7.

12. Balan K. Modified Hodge test and remodified Hodge test for carbapenemase detection: Better indicator. Indian J Appl Res 2013;3:279-80.

13. Vasoo S, Cunningham SA, Kohner PC, Simner PJ, Mandrekar JN, Lolans $\mathrm{K}$, et al. Comparison of a novel, rapid chromogenic biochemical assay, the carba NP test, with the modified Hodge test for detection of carbapenemase-producing gram-negative bacilli. J Clin Microbiol 2013;51:3097-101.

14. Chauhan K, Pandey A, Asthana AK, Madan M. Evaluation of phenotypic tests for detection of Klebsiella pneumoniae carbapenemase and metallo-beta-lactamase in clinical isolates of Escherichia coli and Klebsiella species. Indian J Pathol Microbiol 2015;58:31-5.

15. ElMasry SA, Ammar RA, Saber SM. Phenotypic and molecular characterization of imipenem resistant Pseudomonas isolates. Life Sci J 2012;9:377-83.

16. Dortet L, Poirel L, Nordmann P. Rapid detection of carbapenemase-producing Pseudomonas spp. J Clin Microbiol 2012;50:3773-6. 